\title{
DESENVOLVIMENTO DE PRODUTOS DE FORMA EFICIENTE NAS ORGANIZAÇÕES ATUAIS
}

\section{DEVELOPING PRODUCTS EFFICIENTLY IN ORGANIZATIONS}

\author{
M. L. da CRUZ ${ }^{1, *}$, A. M. O. SIQUEIRA ${ }^{2}$, I. IASBISK ${ }^{3}$, J. DAMATO NETO $^{4}$ \\ 1,*Universidade Federal de Viçosa, DEQ, Viçosa, MG, Brasil, marlonsengprod@ gmail.com \\ ${ }^{2}$ Universidade Federal de Viçosa,, DEQ, Viçosa, MG, Brasil, antonio.siqueira@ufv.br \\ ${ }^{3}$ Presidente Antônio Carlos Foundation, Ubá, MG, Brasil, coordenacaoengenhariacivil@ubafupac.com.br \\ ${ }^{4}$ Presidente Antônio Carlos Foundation, Ubá, MG, Brasil, josedamato@ yahoo.com.br
}

\begin{tabular}{|c|}
\hline A R T I C LE I NFO \\
\hline $\begin{array}{l}\text { Article history: } \\
\text { Received 2017-12-21 } \\
\text { Accepted 2018-06-30 } \\
\text { Available online 2018-10-31 }\end{array}$ \\
\hline $\begin{array}{l}\text { palavras-chave } \\
\text { Desenvolvimento } \\
\text { Produtos } \\
\text { Serviços } \\
\text { Clientes } \\
\text { Organização }\end{array}$ \\
\hline $\begin{array}{l}\text { keywords} \\
\text { Development } \\
\text { Products } \\
\text { Services } \\
\text { Organization }\end{array}$ \\
\hline
\end{tabular}

\begin{abstract}
A B S T R A C T
Due to market and technological competition, today's organizations they need tools that yield sufficient benefits for their growth. The objective of this research is to present the importance of product development efficient in today's organizations. For this, it is considered that products are developed to meet the needs. Therefore, it should be considered that customers have a perception of value and different costs of each other's products and also of the organization. Then it is necessary to market research, which is the responsibility of Marketing, which provides the information needed for product development. At this stage, product ideas are generated and developed. After being executed and linked to the market, the whole cycle must be managed the longer the product, the better for the organization. The use of tools such as 4P's (product, price, promotion and square) assist in making decisions about what, when, how much and where to produce that will ensure for the production and, mainly to the clients, improvement of the quality of the offered products. Efficient product development results in better products at lower costs, to be produced and intended for the consumer, increased profits, sales, customer loyalty and new ones.
\end{abstract}

R E S U M O

Devido à concorrência mercadológica e tecnológica as organizações atuais necessitam de ferramentas que resultem em benefícios suficientes para o crescimento delas. O objetivo da pesquisa é apresentar a importância do desenvolvimento de produtos de forma eficiente nas organizações atuais. Para isso, considera-se que produtos são desenvolvidos para atender às necessidades. Portanto, deve-se considerar que os clientes têm percepção de valor e custo diferentes dos produtos uns dos outros e também da organização. Então é necessária a pesquisa de mercado, de responsabilidade do Marketing que, proporciona as informações necessárias para o desenvolvimento de produtos. Nessa etapa, ideias de produtos são geradas e desenvolvidas. Após serem executadas e laçadas ao mercado, deve-se gerenciar todo o ciclo de vida dos produtos, que quanto mais prolongado for, melhor para a organização. A utilização de ferramentas, como, por exemplo, os 4P's (produto, preço, promoção e praça) auxiliam na tomada de decisões sobre o que, quando, quanto e onde produzir que garantirão para a produção e, principalmente aos clientes, melhoria da qualidade dos produtos ofertados. $O$ desenvolvimento de produtos eficiente resulta em produtos melhores, com menores custos, rápidos de serem produzidos e destinados ao consumidor, aumento dos lucros, das vendas, fidelização de clientes e conquista de novos. 


\section{INTRODUÇ̃̃̃O}

O desenvolvimento tecnológico atingiu, atualmente, um crescimento jamais alcançado. $\mathrm{O}$ modo produtivo automatizado substituiu o mecanizado que substituiu o manual devido às duas Revoluções Industriais, às duas Grandes Guerras Mundiais e a outros fatos históricos que marcaram a transição entre esses três modelos produtivos. Com o advento da Era Informacional, maximizou-se a concorrência mercadológica devido à inovação tecnológica. Infinitas informações tornaram-se disponíveis a todos em qualquer lugar, a qualquer momento. Portanto, a preocupação com o cliente tornou-se fator decisivo e um diferencial competitivo para as organizações atuais. Por isso, houve a necessidade de aperfeiçoamento de diversos conhecimentos e equipes multidisciplinares. Todo processo inicia-se no marketing para entender e atender os clientes. Nesta etapa são desenvolvidas ideias geradas a partir de necessidades e/ou oportunidades observadas e/ou criadas, que passarão por processos de triagem. Nessa fase inicia o contato entre organização e cliente.

Várias outras áreas organizacionais também são responsáveis para com o cliente, tanto em forma de produtos quanto em serviços. Por exemplo, a qualidade deve atender aos prérequisitos do público-alvo, que foi definido na primeira fase de todo o processo. Questões como resistência, fluidez, temperatura, densidade, cor, dimensões entre outras características são consideradas. A produção deve transformar "inputs" que são as entradas no processo, como por exemplo, matéria-prima, mão de obra, maquinário, dentre outros, em "outputs" que são as saídas ou resultados, como, por exemplo, produtos finais, prontos para comercialização e consumo. A manutenção deve manter os equipamentos, máquinas e instalações para haver produção. A logística deve entregar os produtos finais aos clientes corretos, na quantidade e momento certos, a um custo viável compatível com o mercado e com o que os clientes estão dispostos a pagar. Enfim, a organização constitui-se de inúmeros setores interdependentes para atender aos seus respectivos clientes, tanto internos que são os colaboradores, quanto externos que são os clientes e consumidores.

No entanto, há uma área antecedente à produção e posterior ao marketing, denominada desenvolvimento de produtos que também é de fundamental importância para o progresso geral da organização. Esta é uma fase preparatória para o operacional, é aquela que embasa o planejamento da produção. É o elo entre o marketing e a produção, responsável por converter e conciliar interesses dos clientes em produtos finais em restrição das limitações financeiras, humanas, tecnológicas, ambientais, sociais, políticas, dentre outras da organização. É de suma importância para as organizações, pois amplia a participação da empresa em seu nicho de mercado e a egressa em outros, resultando em aumento das receitas através do aumento das vendas com qualidade proporcionando ao cliente o melhor produto da melhor forma possível. Além disso, contribui para a valorização de produto, marca, tecnologias e "know-how" de todos os envolvidos nos processos de criação, produção e logística. Possibilita saúde organizacional prolongando ciclos de vida dos produtos. O desenvolvimento de produtos é uma função estratégica empresarial que auxilia em tomadas de decisões sobre o que produzir, em qual quantidade, de que modo, em qual momento, e qual o público-alvo a ser atendido. Portanto, entender essa função é essencial para planejar e agir antecipadamente, de forma ativa e não reativa ao mercado. Assim, minimizam-se e até evitam-se problemas de insatisfação de clientes através da qualidade de produtos e serviços. Para isso, deve ser flexível o suficiente para lidar com imprevistos de demanda, que são naturais. Uma particularidade dessa área é ela estar presente em todos os níveis departamentais e hierárquicos da empresa. Por exemplo, o marketing deve desenvolver métodos para análise do consumidor; A qualidade, métodos para parametrização de suas funções; A produção, layouts para sua execução; A manutenção, sistemas de controle de paradas de máquinas e as respectivas causas; A logística, sistemas de rotas. Essas etapas desenvolvem produtos ou serviços para executarem suas atividades. Portanto, considera-se que todos os produtos de cada processo surgiram devido ao desenvolvimento sistematizado deles, ratificando que o desenvolvimento de produto se torna uma das etapas principais da organização.

Enfim, planejar corretamente antes de executar é essencial para evitar e superar prejuízos, além de aperfeiçoar processos, produtos e serviços. É necessário atentar às competitividades tecnológicas e mercadológicas para traçar e alcançar metas da organização. O objetivo do presente trabalho é apresentar a importância do desenvolvimento de produto de forma eficiente nas organizações atuais.

\section{CONCEITUAÇÃO E CLASSIFICAÇÃO DAS NECESSIDADES HUMANAS}

Desde os primórdios da humanidade, há busca incessante por satisfazer às necessidades humanas, sejam elas físicas ou psicológicas. Para isso, elas devem ser entendidas para então criar soluções que a satisfaça. Após, analisam-se e escolhem-se uma ou mais dessas soluções para ser desenvolvida. Assim atende-se ao público-alvo da melhor maneira possível. Essas necessidades são classificadas em cinco grupos, de acordo com Maslow. Ele as hierarquiza em forma de pirâmide, do topo para a base: "básicas, segurança, associação, status e autorrealização" (LACOMBE, 2009, p. 130). As necessidades básicas são as fisiológicas, as de segurança se referem aos riscos de agressões físicas ou psicológicas, as de associação indica o egresso em grupos sociais, as de status, ao sentimento de importância própria perante um ou vários grupos de pessoas, e as de autorrealização se referem ao desejo de alcance de metas pessoais. Todas são inter-relacionadas e, considerando a análise da base para topo, cada uma é pré-requisito da anterior. Por exemplo, para atender as necessidades de autorrealização, devese atender às de status, que, por sua vez, deve-se atender às de associação e assim por diante.

\section{TIPOS DE PRODUTOS}

Há uma infinidade de produtos atualmente resultantes do desenvolvimento das necessidades humanas e do consequente desenvolvimento tecnológico. Portanto são agrupados para estudos de mercado e consumidor.

Podem-se classificar os produtos em: Produtos de rotina. São produtos de baixo valor e custo para o cliente e que envolvem pouco risco.

Produtos alavancados. São produtos de alto valor e custo para o cliente, mas que envolvem pouco risco de fornecimento porque muitas empresas os fabricam. Produtos estratégicos. São produtos de alto 
valor e custo para o cliente e que envolvem alto risco. Produtos gargalo. São produtos de baixo valor e custo para o cliente, mas que envolvem algum risco (KOTLER e ELLER, 2006, p. 217).

É importante ressaltar que atualmente há a preocupação ambiental e seus impactos. Portanto, há maior necessidade de fabricar produtos sustentáveis, ou seja, produtos que causam menos impactos negativos e mais positivos. Esses produtos auxiliam na manutenção e equilíbrio do meio ambiente.

\section{DETERMINAÇÃO DO CICLO DE VIDA DOS PRODUTOS}

Para desenvolver algum produto, considera-se o planejamento do ciclo de vida dele, ou seja, deve-se prever quando ele será inserido no mercado, quando ele alcançará estabilidade, quando será o seu declínio e, por fim, quando será o momento em que ele deverá ser retirado do mercado. Podem-se citar vários aspectos que influenciam o ciclo de vida do produto: o volume de vendas, tipo de consumidores, tipo de mercado, economia, produtos concorrentes, dentre outros. De acordo com Martins e Laugeni (2005) todo e qualquer produto tem um início, meio e fim, ou seja, um ciclo de vida. Uns são maiores, outros menores. Alguns já são desenvolvidos com previsão de fim, outros não. Segundo Carpes Junior (2014) o ciclo de vida dos produtos diminui devido ao descarte antecipado, ao excesso de manutenção, devolução não esperada, uso prolongado dentre outros.

Podem-se classificar em quatro fases o ciclo de vida dos produtos, conforme:

Introdução: é a fase inicial da vida do produto, caracterizada por baixo volume de vendas, baixo volume de produção, pedidos sob encomenda e sob medida, produção em pequenos lotes. Muitos produtos não passam dessa fase. Crescimento: o produto começa a firmar-se no mercado, aumenta a demanda e alteram-se os processos produtivos. A empresa procura obter maior volume de produção pela padronização de partes e componentes, automatização de processos, linhas seriadas, fabricação para estoque. Maturidade: há estabilização na demanda e nos processos industriais. Geralmente o produto já atingiu alto grau de padronização. Declínio: demanda decrescente. O produto passa a perder participação no mercado. A empresa deve decidir entre retirá-lo da linha de produção e esperar que ele tenha morte natural (MARTINS e LAUGENI, 2005, p. 73).

\section{GERAÇÃO DE IDEIAS PARA DESENVOLVIMENTO DE PRODUTOS}

"A capacidade de criar novas ideias de produtos não precisa ser restrita a poucas pessoas criativas ou designers talentosos todos têm uma imaginação" (MORRIS, 2001, p. 12). Basta cada um exercitar essa capacidade em raciocínios críticos e detalhados.

Uma das áreas do conhecimento que mais se aproxima do conceito de novas ideias é a engenharia. Logo, engenhosidade é uma característica da Engenharia definida por Barbosa Filho (2009, p. 84) como "a habilidade de realizar associações, combinando ideias e conceitos, ampliando a visão sobre algo ou a realizando de um ângulo diferente. É a engenhosidade, a habilidade central de um engenheiro.” Gurgel (2008, p. 92) afirma que "um produto é considerado criativo à medida que reestruturamos nosso atual universo de conhecimentos e de hábitos associados à utilização de objetos". Segundo (BAXTER, 2003, p. 51) "a criatividade é o coração do design, em todos os estágios do projeto." Para o mesmo autor, "a criatividade pode ser estimulada seguindo determinadas etapas: inspiração inicial, preparação, incubação, iluminação e verificação. Ou seja, o desenvolvimento da criatividade inicia com alguma inspiração, desejo, motivação sobre determinado assunto. Após, a ideia é preparada, ou seja, é planejada com mais detalhes. Em seguida, é amadurecida, mais detalhada, é iluminada, clareada e pronta para ser executada. Então ela é verificada e revisada. Os erros são corrigidos antes da implantação e oportunidades de melhorias são analisadas e executadas.

As melhores ideias são geradas por uma equipe
multidisciplinar, envolvendo marketing,
desenvolvimento de produto e engenharia de
produção. Essas ideias devem ser convertidas em
especificações de projeto, para orientar o
desenvolvimento e fornecer diretrizes para controlar
a qualidade desse desenvolvimento (BAXTER, 2003,
p. 88).

\section{O PROCESSO DE DESENVOLVIMENTO DE PRODUTOS PROPRIAMENTE DITO}

\begin{abstract}
O Processo de Desenvolvimento de Produtos é a forma pela qual a empresa organiza e gerencia o desenvolvimento de produto, determina a obtenção de vantagens competitivas e constitui um pontochave dentro de qualquer empresa que busca a liderança em seu setor de atuação (NEUMANN, 2015).
\end{abstract}

O processo de desenvolvimento de produtos não é algo simples. Portanto é um desafio para os que se envolvem nessa atividade. Há inúmeras variáveis controláveis e não controláveis. A exemplo das primeiras, pode-se controlar o tipo de matériaprima que será utilizada para fabricar o produto e as dimensões dos componentes deles. Já as segundas, pode-se citar a mudança da economia e o surgimento de um público-alvo não esperado. Por isso, deve-se planejar detalhadamente cada etapa. O desenvolvimento depende diretamente do planejamento do produto. Segundo Baxter (2003) iniciar um desenvolvimento sem um eficaz planejamento é começar algo sem saber quais serão os resultados finais. Dentre vários estágios desta fase, (BAXTER, 2003) afirma que os iniciais são cruciais no processo de desenvolvimento de produtos. Ainda segundo Baxter (2003, p. 122) "o planejamento do produto inclui: identificação de uma oportunidade, pesquisa de marketing, análise dos produtos concorrentes, proposta do novo produto, a elaboração da especificação da oportunidade e a especificação do produto." Assim como tudo tem origem, função (ões) e finalidade (s), com esta área não é diferente.

Portanto, "o desenvolvimento de novos produtos é uma atividade importante e arriscada" (BAXTER, 2003, p. 7). Segundo (BAXTER, 2003, p. 21) "o desenvolvimento de novos produtos é um problema multifatorial: o sucesso ou fracasso depende de muitos fatores, tais como: simpatia dos consumidores, aceitação dos distribuidores, facilidades de fabricação, durabilidade e confiabilidade do produto." É 
importante ressaltar que desenvolver novos produtos é muito importante ao mesmo tempo, arriscada. São inúmeras às vezes em que ocorrem mudanças e reprojetos são necessários. Cada alteração é vital para o objetivo inicial e primordial que é atender aos clientes.

Todo produto deve ser funcional, de fácil utilização, considerar os aspectos ergonômicos envolvidos, ter estética, comandos auto-explicativos - como os comandos dos painéis dos automóveis - ser compatível com as preocupações de preservação do meio ambiente, e seu projeto apoiar-se em tecnologia conhecida e ter contato com a colaboração de equipes multifuncionais, no sentido de ser facilmente fabricado e montado (MARTINS E LAUGENI, 2005, p. 70).

Os produtos podem ser classificados, segundo Carpes Junior (2014) em quatro: tangível, genérico, esperado e ampliado. Do tangível para o ampliado são acrescidos valores ao produto ao cliente proporcionando a este determinado grau de satisfação.

\section{IMPORTÂNCIA DOS 4 P'S PARA O DESENVOLVIMENTO DE PRODUTOS}

Os clientes estão inseridos em um determinado mercado definido pelo marketing. São eles que ditam qual o tipo e volume de demanda em função de inúmeras questões, tais como financeiras, temporais, culturais, dentre outras. Baxter (2003, p. 130) afirma que "a demanda do mercado refere-se à procura, pelo mercado, de produtos ou características do produto que ainda não são oferecidos pela sua empresa." Uma das estratégias para melhorar o atendimento dessa demanda é a utilização dos 4P's. "Produto, preço, praça e promoção (os quatro P's) são as ferramentas de marketing que, se utilizadas em conjunto, possibilitam que um produto seja vendido da forma mais eficaz" (MORRIS, 2011, p. 162).

De acordo com Paiva e Neves (2008) os 4P's do produto significam produto, preço, promoção e praça. Produto significa as características do produto em si como, por exemplo, design, dimensões, qualidade. Preço se refere à questão financeira, como preço, condições e prazos de pagamento e financiamento. Promoção de refere aos esforços para promover o produto, ou seja, divulgar, torná-lo público, por exemplo, publicidade, marketing. E, por último, praça ou distribuição significa os locais físicos por onde o produto passará, por exemplo, estoques, transportes, locais.

\section{IMPORTÂNCIA DO DESENVOLVIMENTO DE PRODUTOS DE FORMA EFICIENTE PARA A PRODUÇÃO}

A produção depende dos resultados do desenvolvimento de produtos para produzir da melhor forma possível, assim como o desenvolvimento de produtos depende do planejamento que depende do marketing para que cada um desempenhe sua função o melhor possível.

Martins e Laugeni (2005, p. 2) definem a função produção como sendo "entendida como o conjunto de atividades que levam à transformação de um bem tangível em outro com maior utilidade, acompanha o homem desde sua origem." Até atingir o modo como se encontra atualmente, houve uma série de mudanças ao longo do tempo. A produção artesanal foi um dos primeiros modos produtivos que existiu e não existe mais porque "começou a entrar em decadência com o advento da Revolução Industrial” (MARTINS e LAUGENI, 2005, p. 2). A partir daí houve a introdução de máquinas e automação devido à demanda por maior volume e qualidade de produtos.

\section{IMPORTÂNCIA DO DESENVOLVIMENTO DE PRODUTOS DE FORMA EFICIENTE PARA A QUALIDADE DOS PRODUTOS}

Todas as propriedades ou características de um produto ou serviço relacionados com sua capacidade de satisfazer às necessidades explícitas ou implícitas dos que o utilizam, isto é, dos clientes, sem prejudicar os que são afetados pelo uso do produto ou serviço (LACOMBE, 2009, p. 255).

"A qualidade de um produto tem muitos significados diferentes para diferentes pessoas" (BAXTER, 2003, p. 207).

Para o consumidor, a prova da qualidade do produto é seu desempenho quando submetido a golpes, sobrecargas e quedas. Ou seja, o produto deve suportar não apenas variações no processo produtivo, mas também as mais difíceis de uso sem apresentar defeitos (MARTINS e LAUGENI, 2005, p. 72).

De acordo com (KOTLER e KELLER, 2006, p. 145) "qualidade total é o segredo para criar valor e satisfazer o cliente." "Quando as qualidades de um produto não são satisfatórias, as expectativas do consumidor são frustradas." (CARPES JUNIOR, 2014, p. 10). Ainda, segundo Carpes Junior (2014) alguns desses defeitos são: problemas no controle de qualidade, no projeto do produto ou avisos ou instruções erradas. Destacam-se várias características no que tange o termo qualidade, como por exemplo, funcionalidade, segurança, estética, economia, sustentabilidade, ergonomia, produtividade e operacionalidade.

Para Lacombe (2009) as consequências de um bom programa de gerenciamento de qualidade são melhorias no projeto do produto, no produto em si, no processo de fabricação, nas características psicológicas dos empregados, na aceitação do mercado, na assistência técnica prestada ao consumidor, além de redução dos custos operacionais e dos serviços prestados ao consumidor, das perdas do processo de fabricação e consequentemente das reclamações.

\section{CONSIDERAÇÕES FINAIS}

O desenvolvimento de produtos de forma eficiente nas organizações, utilizando corretamente as informações sobre os 4P's, ou seja, sobre o melhor produto, com menor preço, ofertado no mercado com qualidade e distribuído da melhor forma possível, resulta em melhor qualidade de processos de produção e consequentemente melhor qualidade dos produtos e dos serviços. Ou seja, há redução de custos de planejamento, desenvolvimento, produção e logística e aumento de lucros. As expectativas dos consumidores são superadas e as necessidades deles são atendidas. Através disso, as organizações mantêm os atuais clientes, conquistam novos clientes e superam as 
concorrências mercadológicas e tecnológicas, com mais facilidade do que as que não possuem um bom desenvolvimento de produtos.

\section{R E F E R E N C I A S}

BARBOSA FILHO, Antonio Nunes. Projeto e desenvolvimento de produtos. São Paulo: Atlas, 2009. 181 p. Disponível em: <http://integrada.minhabiblioteca.com.br/\#/books/9788522464 760/cfi/0>. Acesso em: 20 nov. 2017.

BAXTER, Mike. Projeto de produto: guia prático para o design de novos produtos. 2. ed. São Paulo: Edgard Blucher, 2003. $260 \mathrm{p}$.

CARPES JUNIOR, Widomar P. Introdução ao projeto de produtos. Porto Alegre: Bookman, 2014. 229 p. Disponível em: https://integrada.minhabiblioteca.com.br/\#/books/9788582602 409/cfi/0. Acesso em: 25 nov. 2017.

GURGEL, Floriano do Amaral. Administração do produto. 2. ed. São Paulo: Atlas, 2008. 537 p. Disponível em: https://integrada.minhabiblioteca.com.br/\#/books/9788522472 628/cfi/0. Acesso em: 23 nov. 2017.

KOTLER, Philip; KELLER, Kevin Lane. Administração de marketing. Tradução: Mônica Rosenberg, Cláudia Freire, Brasil Ramos Fernandes. 12. ed. São Paulo: Pearson Prentice Hall, 2006. 750 p.

LACOMBE, Francisco. Teoria geral da administração. São Paulo: Saraiva, 2009. 351 p. Disponível em: https://integrada.minhabiblioteca.com.br/\#/books/9788502089 181/cfi/0. Acesso em: 24 nov. 2017.

MARTINS, Petrônio G.; LAUGENI, Fernando P. Administração da produção. 2 ed. São Paulo: Saraiva, 2005. 562p. Disponível em: https://integrada.minhabiblioteca.com.br/\#/books/9788502089 174/cfi/0. Acesso em: 24 nov. 2017.

MORRIS, Richard. Fundamentos de design de produto. Porto Alegre: Bookman, 2011. 184 p. Disponível em: https://integrada.minhabiblioteca.com.br/\#/books/9788577808 250/cfi/0. Acesso em: 24 nov. 2017.

NEUMANN, Clóvis. Engenharia de produção para concursos. Rio de Janeiro: Elsevier, 2015. 744 p. Disponível em: https://integrada.minhabiblioteca.com.br/\#/books/978-85309-6449-8/cfi/6/2[;vnd.vst.idref=cover.html]. Acesso em: 24 nov. 2017.

PAIVA, Hélio; NEVES, Marcos. Planejamento estratégico de eventos. São Paulo: Atlas, 2008. 192 p. Disponível em: https://integrada.minhabiblioteca.com.br/\#/books/9788522464 531/cfi/0. Acesso em: 24 nov. 2017. 